\title{
Overview of Therapies for Diabetic Macular Edema
}

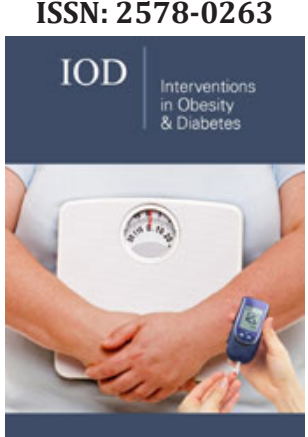

*Corresponding author: Gabriela YCM, Hospital Regional de Alta Especialidad de Ixtapaluca, Zoquiapan, Ixtapaluca, Estado de Mexico, Mexico

\section{Submission: 慍June 04, 2019 \\ Published: 楷 June 14, 2019}

Volume 3 - Issue 1

How to cite this article: Gabriela Y, Italia A R, Gustavo A, Adrian H, Tania V. Overview of Therapies for Diabetic Macular Edema. Interventions Obes Diabetes. 3(1). IOD.000552.2019.

DOI: 10.31031/IOD.2019.03.000552

Copyright@ Gabriela YCM, This article is distributed under the terms of the Creative Commons Attribution 4.0 International License, which permits unrestricted use and redistribution provided that the original author and source are credited.
Gabriela YCM${ }^{1,2 *}$, Italia A Rivera ${ }^{1,3}$, Gustavo AA $^{1,2}$, Adrian Heald ${ }^{4,5}$ and Tania VS ${ }^{1}$

${ }^{1}$ Hospital Regional de Alta Especialidad de Ixtapaluca, State of Mexico, Mexico

${ }^{2}$ School of Medicine, Instituto Politécnico Nacional, Mexico City, Mexico

${ }^{3}$ Centro Interdisciplinario de Ciencias de la Salud, Unidad Milpa Alta, Instituto Politécnico Nacional, Mexico City, Mexico

${ }^{4}$ The School of Medicine and Manchester Academic Health Sciences Centre, United Kingdom

${ }^{5}$ Department of Diabetes and Endocrinology, Salford Royal NHS Foundation Trust, United Kingdom

\begin{abstract}
Diabetic retinopathy is the most common microvascular complication of diabetes mellitus. The direct cause of this irreversible loss of sight is diabetic macular edema, which affects more than 126 million people worldwide. In order to confirm the diagnosis, specific studies, such as fluorescein angiography and optical coherence tomography (OCT), should be carried out to determine the degree and type of edema. We review recent efficacy and safety data for the therapies currently existing to reduce progression and recover vision in Diabetic Macular Edema. In addition, there is the high cost of treatment of macular edema, as well as the risks involved in the application of drugs used for this disease. We therefore decided to carry out this review in order to evaluate the effectiveness of different therapies for the management of diabetic macular edema. The growing controversy over treatment for this complication has led to a constant conflict over what the best therapeutic option for the affected individuals is. Alternatives for supplementation have also been sought out with studies that support the efficacy of their use in treating this disease.
\end{abstract}

Keywords: Anti-VEGF; Diabetic Retinopathy; Lutein; Macular Edema; Oxidative-Stress; Therapies

Keywords: OCT: Optical Coherence Tomography; DR: Diabetic Retinopathy; DME: Diabetic Macular Edema; VEGF: Vascular Endothelial Growth Factor; DRCR: Diabetic Retinopathy Clinical Research; ILM: Internal Limiting Membrane

\section{Introduction}

In 2017, the number of diabetics in the world was more than 451 million [1]. In Mexico, there are more than 12 million people diagnosed with Type 1 or 2 Diabetes [2]. Diabetic retinopathy (DR) is the most common microvascular complication of diabetes [3] and occurs in $47.26 \%$ of the population with type 1 diabetes mellitus, and in $26.49 \%$ of type 2 diabetics [4]. Diabetic macular edema (DME) is the leading worldwide cause of acquired loss of vision in middle aged, and therefore economically active, people [5]. It affects one in 15 people with diabetes, resulting in more than 20 million cases worldwide [6]. Studies conducted on the population of Mexico City reported that the proportion of diabetics with DME who have visual impairment at the time of diagnosis is 69.4\% [7]. Certain studies in America report a prevalence of Diabetic Retinopathy in $33.3 \%$ of patients $(29.9 \%$ with Non-Sight Threatening Retinopathy and 3.4\% with Sight Threatening Retinopathy, more than half of which suffer from macular edema) [8,9], (Figure 1).

The presence of retinal edema is defined as an increase in interstitial fluid at tissue level, which causes a thickening of the central retina (macula). This fluid increase can be intracellular or extracellular, which induces an alteration of the external blood-retinal barrier [10]. Among the factors that predispose people with diabetes to visual damage are hyperglycemia and retinal ischemia. Through a series of pathophysiological processes, there is stimulation of the synthesis and secretion of vascular endothelial growth factor (VEGF) and interleukin 6 (IL-6) [11]. These factors act directly on the tight junctions of endothelial cells, decreasing protein content or increasing phosphorylation; altering the paracellular permeability [12]. 


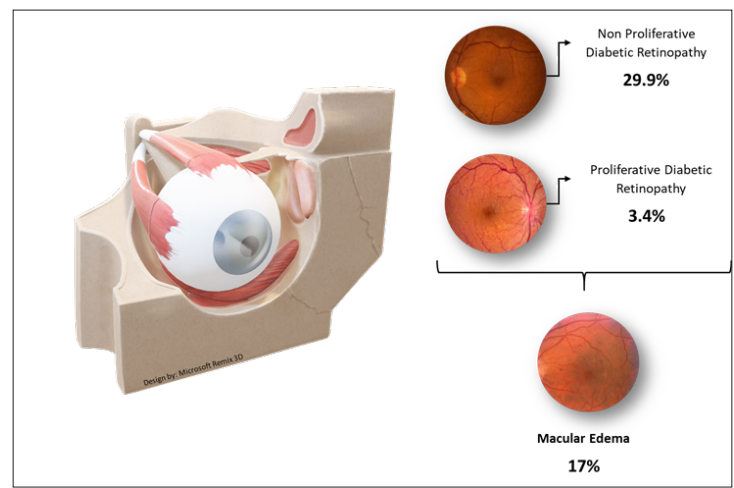

Figure 1: Prevalence of diabetic retinopathy (non-proliferative/ proliferative retinopathy).

\section{Treatment}

Existing therapies can be divided into 3 types: Pharmacological by administering anti-VEGF drugs (Ranibizumab 0.3 or $0.5 \mathrm{mg}$, Bevacizumab $1.25 \mathrm{mg}$, or Aflibercept, $2 \mathrm{mg}$ ) or corticosteroids (Dexamethasone Implant, Fluocinolone Acetonide and Triamcinolone Acetonide); Interventions, such as vitrectomy and the application of conventional or pulsed lasers; Emerging therapies of natural origin, such as lutein in capsules or pycnogenol (French pine extract) [13], (Figure 2). According to the International Council of Ophthalmology's Clinical Guidelines for the management of ocular pathology of the diabetic: The treatment of macular edema in high-resource countries, according to the severity of the retinal manifestations and the clinical picture, is as follows:

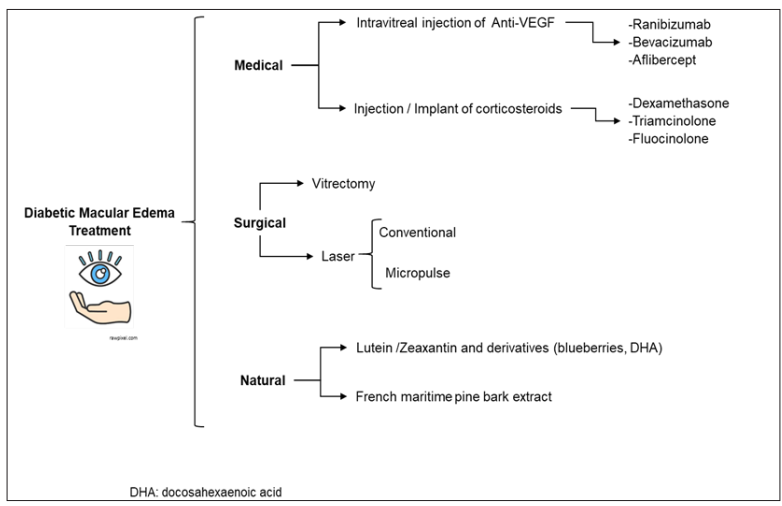

Figure 2: Flow chart of the main treatments for diabetic macular edema.

a. DME with central damage and good visual acuity (better than 20/30): Careful follow-up with anti-VEGF treatment or laser photocoagulation with anti-VEGF, if necessary.

b. DME with central commitment and associated vision loss (20/30 or worse): Intravitreal anti-VEGF treatment. For persistent thickening of the retina, consider laser treatment after 24 weeks. Treatment with intravitreal triamcinolone may also be considered, especially in pseudophakic eyes. For low/medium resource countries, focal laser is preferred if intravitreal injection of antiVEGF drugs is not available or if a monthly follow-up is not possible [14].

\section{Focal macular laser}

For many years the main treatment for DME was focal laser, sometimes supplemented with corticosteroid injections. Laser treatment reduced the risk of moderate visual loss in approximately $50 \%$ of the patients, but only $3 \%$ of the eyes displayed improved vision ( $<3$ lines of visual acuity) and a substantial proportion of people had no response to treatment [15]. In the study conducted by Mitchell et al. [16], in which 345 patients with DME participated, a reduction of micrometers $(\mu \mathrm{m}$,) of the edema measured by optical coherence tomography (OCT) was observed. In the laser monotherapy there was a decrease of $-61.3 \mu \mathrm{m}(\mathrm{p} \leq 0.001)$. However, in the same study, an anti-VEGF drug was also administered to another group and a further group underwent a combination of both therapies. It was shown that the combination of laser therapy with anti-VEGF was more effective than laser monotherapy on its own, showing a decrease of $-128.3 \mu \mathrm{m}(\mathrm{p} \leq 0.001)$. A significantly higher proportion of patients had an initial BCVA (Visual Corrected Acuity) score of $\geq 15$ (Snellen equivalent 20/500) and a subsequent score of $>73$ (Snellen equivalent of 20/40) with single laser $(8.2 \%$ and 23.6\%); Ranibizumab (22.6\% and 53\%, respectively) and Ranibizumab+laser (22.9\% and $44.9 \%$ ) [16]. It was concluded that laser treatment combined with an anti-VEGF drug is more effective than laser therapy on its own in rapidly improving and maintaining Visual Acuity in patients with visual impairment due to Diabetic Macular Edema [17], (Table 1). 


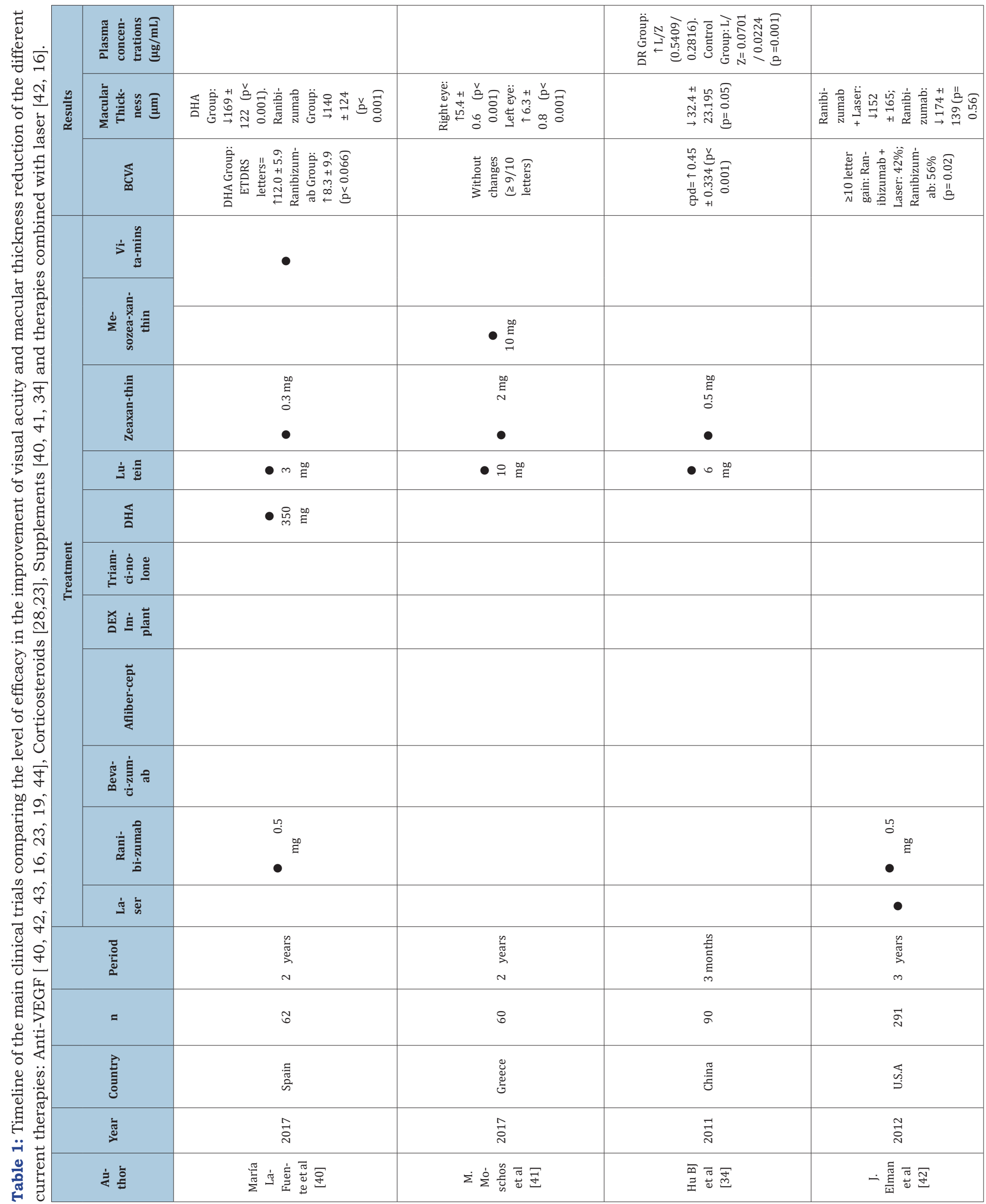




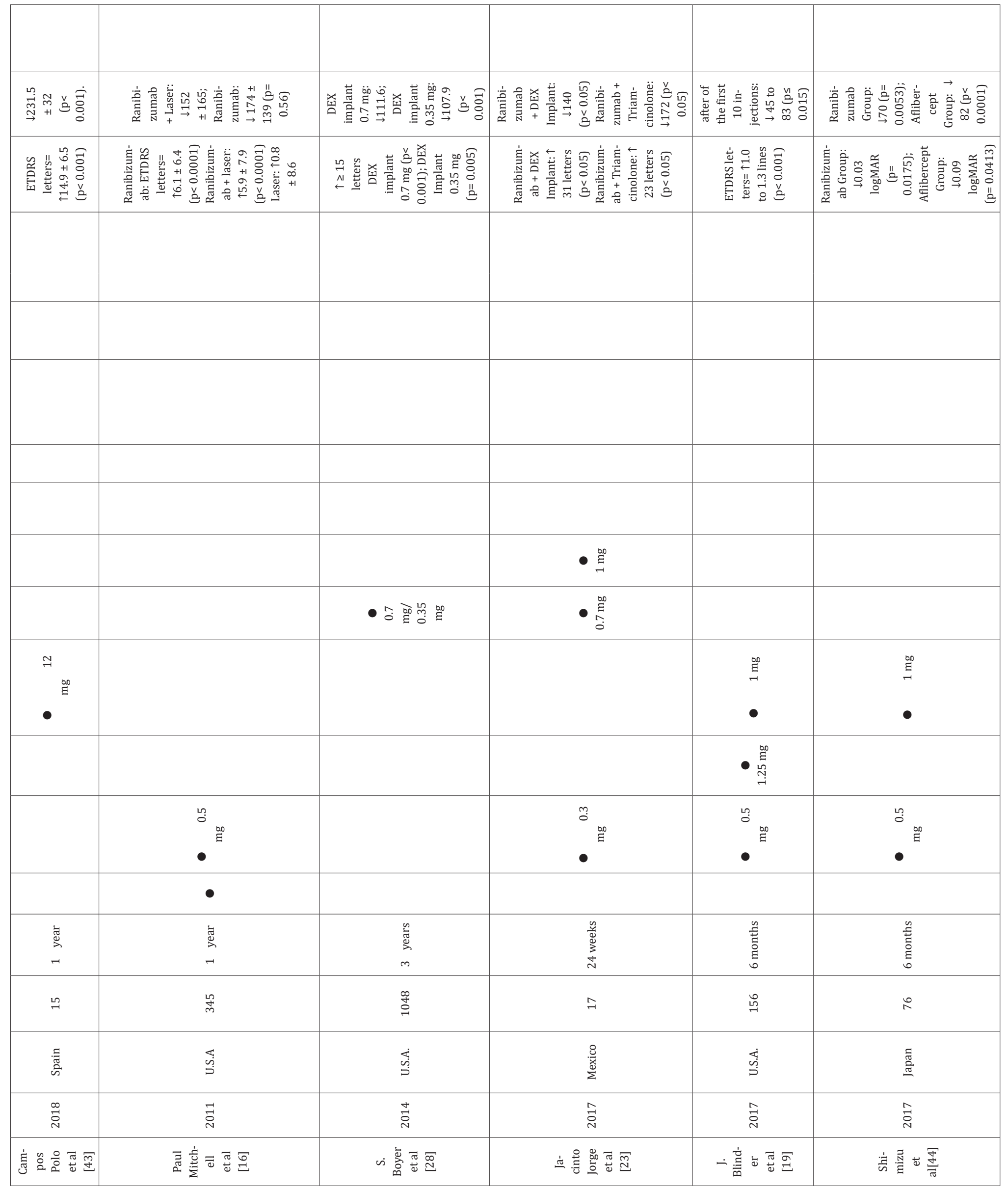




\section{Anti-VEGF injections}

Anti-VEGF treatments achieved great popularity because they act by reducing angiogenesis and vascular permeability, processes that cause the regression of diabetic neovascularization [18]. Blinder et al. [19], in a retrospective study with 156 patients who received more than 3 injections of anti-VEGF (627 injections of Bevacizumab, 594 of Ranibizumab and 1 of Aflibercept), found that the percentage of patients with BCVA of 20/40 or better and macular thickness $<250 \mu \mathrm{m}$ increased from $16.4 \%$ to $38.9 \%$ after the first 10 injections. In addition, more than $15 \%$ of people had achieved BCVA $20 / 40$ and $22 \%$ decreased their macular thickness. However, the results of this study conclude that a significant percentage of patients treated with intravitreal anti-VEGF did not achieve 20/40 or more visual acuity after anti-VEGF injection, possibly because this medication does not inhibit all inflammatory cytokines of the EMD [19] (Table 1). In the VISTA and VIVID studies, a significant improvement in visual acuity was reported $(\mathrm{p} \leq 0.001)$ compared to the laser. The proportion of eyes that gained $\geq 15$ letters from the beginning in week 148 was $42.9 \%$ with 2 mg of aflibercept every 4 weeks, $35.8 \%$ with a dose of $2 \mathrm{mg}$ every 8 weeks of Aflibercept and $13.6 \%$ with laser alone ( $\mathrm{p} \leq 0.001)$. [20].

The Diabetic Retinopathy Clinical Research Network (DRCR Protocol) compares the effects of the three antiangiogenic agents and reports that there are no significant differences between them; concluding that all three intravitreous anti-VEGF agents have acceptable ocular and systemic safety profiles to support their use as first-line treatment for DME, when accessible, over observation, focal/grid laser or corticosteroids with or without concomitant laser treatment [21] Patients who respond suboptimally to treatment with anti-VEGF may need additional therapy with corticosteroids [22]. Jacinto et al. [23]; affirms that this combination improved visual acuity, significantly reduced central macular thickness and did not significantly increase intraocular pressure in patients with diabetic macular edema. In their clinical trial, 17 patients received Anti VEGF injections and corticosteroid implants for 24 weeks. An improvement in 28-letter $(\mathrm{p} \leq 0.05)$ visual acuity was observed on average, accompanied by a reduction of $158 \mu \mathrm{m}(\mathrm{p} \leq 0.05)$ in central macular thickness and an increase in intraocular pressure of $1 \mathrm{mmHg}$. One of the patients underwent cataract surgery and one participant had increased intraocular pressure of more than $10 \mathrm{mmHg}$, which was controlled with the addition of an ocular hypotensive [23].

\section{Corticosteroids}

Corticosteroids are drugs that inhibit macrophages that release angiogenic growth factors, in addition to diminishing the expression of the major histocompatibility complex (MHC-II) in the superficial layers of the retina $[24,25]$. However, their use has been decreasing due to concerns about cataract formation and increased intraocular pressure [26]. Studies report that up to a third of patients treated with intravitreal implants of Dexamethasone (DEX) achieve a vision of 20/40 after their first implant, and the benefit of the treatment is maintained in the long term [27]. This implant has shown efficacy and has had an acceptable safety profile as reported by Boyer et al. [28]. In this study, 1048 patients received a Dexamethasone implant of $0.7 \mathrm{mg}, 0.35 \mathrm{mg}$ and a placebo group for 3 years. The percentage of patients with $\geq 15$-letter improvement in BCVA from baseline at study was greater with DEX implant $0.7 \mathrm{mg}(22.2 \%)$ and DEX implant $0.35 \mathrm{mg}(18.4 \%)$ than the sham implant $12.0 \%$; $\mathrm{p} \leq 0.018)$. Mean average reduction in macular thickness from baseline was greater with the DEX implant $0.7 \mathrm{mg}(-111.6 \mu \mathrm{m})$ and DEX implant $0.35 \mathrm{mg}(-107.9 \mu \mathrm{m})$ than sham $(-41.9 \mu \mathrm{m}, \mathrm{p} \leq 0.001)$. Rates of cataract-related adverse effects in phakic eyes were $67.9 \%$ (DEX implant $0.7 \mathrm{mg}$ ), 64.1\% (DEX implant $0.35 \mathrm{mg}$ ), and $20.4 \%$ in the sham group. Increases in intraocular pressure were usually controlled with medication or without therapy; only 2 patients $(0.6 \%)$ in the DEX implant $0.7 \mathrm{mg}$ group and $1(0.3 \%)$ in the DEX implant $0.35 \mathrm{mg}$ group required trabeculectomy [28].

There is scientific evidence that corticosteroids are a good option for persistent macular edema, as in the study by McCluskey et al. [29] injections of $0.2 \mu \mathrm{g} /$ day fluocinolone acetonide [FAc] were performed intravitreal implant in patients with persistent or recurrent DME and a reduction of the macular volume was observed in $89 \%$ of the eyes, with a statistically significant $(p \leq 0.001)$. The average central retinal thickness reduction for all 18 eyes was statistically significant, decreasing from $444 \mu \mathrm{m}$ at baseline to $359 \mu \mathrm{m}$ after the FAc implant ( $\mathrm{p} \leq 0.001$ ). In $90 \%$ of eyes, visual acuity was stable throughout the follow-up period. Despite being a safe therapy, it is necessary to take into account side effects of intravitreal steroids, such as increased intraocular pressure and cataracts. Therefore, it is necessary to administer them with caution and make periodic revisions to avoid their occurrence (Table 1).

\section{Vitrectomy}

People with diabetes very frequently have vitreomacular traction (TVM) or epiretinal membranes (ERM) on the macula. The pull-on retinal vessels increase their permeability and produces tractional diabetic macular edema (EMDT). Surgery is reserved for cases in which there is vitreomacular traction, because it is in these cases where vitrectomy is effective [30]. The vitrectomy with internal limiting membrane (ILM) peeling has efficacy for laser-insensitive patients or patients did not respond to anti-VEGF or steroids substances and is the last method for the therapy of refractory DME [31] Gandorfer et al. [32] suggested that ILM peeling led to an earlier and greater decrease of retinal thickness or to an earlier and better VA and can decrease the risk of subsequent ERM formation by eliminating a scaffold for proliferating cells [32].

\section{Lutein supplements}

Emerging therapies of natural origin, due to their antioxidant effect, have been shown to be more effective if they are administered as adjuvant treatment and prophylactically [33]. However, they maintain an efficacy similar to or even greater than that of conventional lasers. Studies have been conducted with Lutein oral supplementation, as reported by Hu et al. [34], in which 30 individuals with Non-Proliferative diabetic retinopathy were treated with $6 \mathrm{mg} / \mathrm{d}$ and zeaxanthin $0.5 \mathrm{mg}$ /day for three months. There was another group of 30 subjects without supplementation and a Control group. Visual acuity and foveal thickness were recorded at the beginning and 3 months after supplementation. 
Significant improvement in visual acuity was observed after the medication, compared to the BCVA baseline ( $F=18,698, \mathrm{p} \leq 0.001)$. Furthermore, the foveal thickness after Lutein and Zeaxanthin supplementation was significantly decreased (mean 286.50 +/134.185, p s 0.05) [34] (Table 1).

\section{Systemic treatment}

Systemic Agents to Treat DME: Hypoglycemic Agents (Insulin Therapy, Thiazolidinediones Biguanides) [35]; Hypolipidemic Agents (Fibrates, Statins) [36]; Antihypertensive Agents (Angiotensin-2 Converting Enzyme Inhibitors, Angiotensin-2 Receptor Blockers) [37]; Antiplatelet Agents [38]; Ruboxistaurin and Somatostatin Derivatives [39]. These types of drugs, used in chronic and autoimmune diseases, have been tested to slow the progression of diabetic retinopathy. However, they require more scientific sustenance to be considered effective options.

\section{Conclusion}

We continue to search for an effective monotherapy to treat central visual loss in those who are found to have Diabetic Retinopathy, complicated by DME. According to the options that exist today, it is concluded that all demonstrate a certain degree of effectiveness, because the mechanism of action is different in each one. However, the administration of combination therapy with antiVEGF [40-44] and laser or with corticosteroids is recommended, as it results in greater improvement of visual acuity and decrease in macular thickness. General recommendations in general for the choice of therapy should be based on three fundamental aspects: the level of visual acuity, the degree of macular thickness and the area of visual commitment. The availability of each drug sometimes results in the choice being what is available to the patient, rather than what is most appropriate. The high costs, application technique and myths around each therapy are some of the limitations that impede their prescription.

\section{Limitation}

More studies are required in which a comparison is made between emerging therapies, such as micropulsed laser vs early administration of Lutein. Finally, emerging therapies, such as the oral administration of supplements that decrease macular degeneration associated with age, plant derivatives and other components, provide a prophylactic and preventive option of great help that should be implemented in a timely manner in all people suffering from diabetic retinopathy, given that prevention of DME must always be the first priority.

\section{Conflict of Interest}

The authors declare that there is no conflict of interest regarding the publication of this paper.

\section{Acknowledgment}

We thank optometrist Edgar Lara Cibrian for contributing for their knowledge in the subject.

\section{References}

1. Cho NH, Shaw JE, Karuranga S, Huang Y, Ohlrogge AW, et al. (2018) IDF diabetes atlas: Global estimates of diabetes prevalence for 2017 and projections for 2045. Diabetes Res Clin Pract 138: 271-281.

2. Shamah LT, Ruiz MC, Rivera DJ, Kuri MP, Cuevas NL, et al. (2017) National survey of health and nutrition of midway 2016, national results cuernavaca Mexico. National Institute of Public Health (MX).

3. Bursell SE, Fonda SJ, Lewis DG, Horton MB (2018) Prevalence of diabetic retinopathy and diabetic macular edema in a primary care-based teleophthalmology program for American Indians and Alaskan Natives. PLOS ONE 13(6): e0198551.

4. Pedro RA, Raul NG, Aida VM, Ramon SA, Antonio MR, et al. (2017) Differences in incidence of diabetic retinopathy between type 1 and 2 diabetes mellitus: A nine-year follow-up study. British Journal of Ophthalmology 101(10): 1346-1351.

5. Ting DS, Cheung GC, Wong TY (2016) Diabetic retinopathy: Global prevalence, major risk factors, screening practices and public health challenges: A review. Clinical \& Experimental Ophthalmology 44(4): 260-277.

6. Gavin ST, Ning C, Rafael S, et al. (2017) Diabetic macular oedema. The Lancet Diabetes \& Endocrinology 5(2):143-155.

7. Ávila AY, Lima GV (2013) Retinopathy that threatens vision in diabetic patients not referred to the ophthalmologist. Medical Gazette of Mexico 149: 624-629.

8. Carrillo ALC, Erika LL, Hernández AC, Martínez CJA (2011) Prevalence of diabetic retinopathy in patients with type 2 diabetes mellitus in Hidalgo, Mexico. Mexican Journal of Ophthalmology 85(3): 142-147.

9. Ding J, Wong TY (2012) Current epidemiology of diabetic retinopathy and diabetic macular edema. Current Diabetes Report 12(4): 346-354.

10. Estevez BN, et al. (2017) Some considerations about diabetic macular edema. University of Medical Sciences, ISSN 1029-3019 MEDISAN 21(5).

11. Funatsu H (2006) Cytokines and pathogenesis of diabetic macular edema. Department of Ophthalmology, Japan 2(4).

12. Förster C (2008) Tight junctions and the modulation of barrier function in disease. Histochemistry and cell biology 130(1): 55-70.

13. Ford JA, Lois N, Royle P, Christine C, Shyangdan D, et al. (2013) Current treatments in diabetic macular oedema: systematic review and metaanalysis. BMJ Open 3: e002269.

14. Taylor H, et al. (2017) Clinical Guidelines for the management of diabetic ocular pathology. International Journal of Ophthalmology.

15. Hernández SL, Aranda SS (2015) Guide for the diagnosis and treatment of diabetic macular edema in right-holders of the ISSSTE. Rev Esp Méd Quir: 321-327.

16. Mitchell P, Bandello F, Schmidt EU, Lang GE, Massin P, et al. (2011) The RESTORE study ranibizumab monotherapy or combined with laser versus laser monotherapy for diabetic macular edema. American Academy of Ophthalmology 118(4): 615-625.

17. Elman MJ, Aiello LP, Beck RW, Bressler NM, Bressler SB, et al. (2010) Randomized trial evaluating ranibizumab plus prompt or deferred laser or triamcinolone plus prompt laser for diabetic macular edema. Ophthalmology 117(6): 1064-1077.

18. Simó R, Hernández C (2008) Intravitreous anti-VEGF for diabetic retinopathy: hopes and fears for a new therapeutic strategy. Diabetology 51(9): 1574-1580.

19. Blinder KJ, Dugel PU, Chen S, Jumper JM, Walt JG, et al. (2017) Anti-VEGF treatment of diabetic macular edema in clinical practice: effectiveness and patterns of use (ECHO Study Report 1). Clinical Ophthalmology 11: 393-401. 
20. Heier JS, Korobelnik JF, Brown DM, Schmidt EU, Do DV, et al. (2016) Intravitreal aflibercept for diabetic macular edema: 148-week results from the VISTA and VIVID studies. Ophthalmology 123(11): 2376-2385.

21. Cai S, Bressler NM (2017) Aflibercept, bevacizumab or ranibizumab for diabetic macular oedema: recent clinically relevant findings from DRCR. net Protocol T. Curr Opin Ophthalmol 28(6): 636-643.

22. Regillo CD, Callanan DG, Do DV, Fine HF, Holekamp NM, et al. (2017) Use of corticosteroids in the treatment of patients with diabetic macular edema who have a suboptimal response to anti-VEGF: recommendations of an expert panel. Ophthalmic Surgery Lasers and Imaging Retina 48(4): 291-301.

23. Jacinto JE, et al. (2017) Combination of antiangiogenic and intravitreal steroids in the treatment of diabetic macular edema. Revista Médica MD $9(1)$.

24. Tokida Y, Aratani Y, Morita A, Kitagawa Y (1990) Production of two variant forms by endothelial cells and shift of their relative levels by angiostatic steroids. The J Biol Chem 265(30): 18123-18129.

25. Stokes CL, Weisz PB, Williams SK, Lauffenburger DA (1990) Inhibition of microvascular endothelial cell migration by beta-cyclodextrin tetradecasulfate and hydrocortisone. Microvasc Res 40 (2): 279-84.

26. Grover DA, Li T], Chong CC (2008) Intravitreal steroids for macular edema in diabetes. Cochrane Database of Systematic Reviews CD005656.

27. Dal G, Raj M (2015) Study compares eylea, lucentis and avastin for diabetic macular edema; American Academy of Ophthalmology; NCT01627249.

28. Boyer DS, Yoon YH, Belfort R, Bandello F, Maturi RK, et al. (2014) Threeyear, randomized, sham-controlled trial of dexamethasone intravitreal implant in patients with diabetic macular edema. American Academy of Ophthalmology 121(10); 1904-1914.

29. McCluskey JD, Kaufman PL, Wynne K, Lewis G (2019) Early adoption of the fluocinolone acetonide (FAc) intravitreal implant in patients with persistent or recurrent diabetic macular edema (DME). Int Med Case Rep J 12: 93-102.

30. Diego RC (2016) Surgery of Diabetic Macular Edema. Vitrectomy Spain. (http://www.doctordiegoruizcasas.com/oftalmology/ocular-surgery/ diabetic-retinopathy-surgery/ diabetic-macular-edema-emd.html)

31. Hu XY, Liu H, Wang LN, Ding YZ, Luan J (2018) Efficacy and safety of vitrectomy with internal limiting membrane peeling for diabetic macular edema: A Meta-analysis. Int J Ophthalmol 11(11): 1848-1855.

32. Gandorfer A, Messmer EM, Ulbig MW, Kampik A (2000) Resolution of diabetic macular edema after surgical removal of the posterior hyaloid and the inner limiting membrane. Retina 20(2): 126-133.

33. Ma L, Yan SF, Huang YM, Lu XR, Qian F, et al. (2012) Effect of lutein and zeaxanthin on macular pigment and visual function in patients with early age-related macular degeneration. Ophthalmology 119 (11): 2290-2297.

34. Hu BJ, Hu YN, Lin S, Ma WJ, Li XR (2011) Application of lutein and zeaxanthin in nonproliferative diabetic retinopathy. Int J Ophtalmol 4(3): 303-306.

35.(1998) UK prospective diabetes study (UKPDS) Group, Intensive blood-glucose control with sulphonylureas or insulin compared with conventional treatment and risk of complications in patients with type 2 diabetes (UKPDS 33). The Lancet 352(9131): 837-853.

36. Chen XR, Besson VC, Palmier B, Garcia Y, Plotkine M, et al. (2009) Neurological recovery-promoting, anti-inflammatory, and anti-oxidative effects afforded by fenofibrate, a PPAR alpha agonist, in traumatic brain injury. Journal of Neurotrauma 24(7): 1119-1131.

37. Vaajanen A, Lakkisto P, Virtanen I, Kankuri E, Oksala O, et al. (2010) Angiotensin receptors in the eyes of arterial hypertensive rats. Acta Ophthalmologica 88(4): 431-438.

38. (1989) The DAMAD Study Group, Effect of aspirin alone and aspirin plus dipyridamole in early diabetic retinopathy. A multicenter randomized controlled clinical trial. Diabetes 38(4): 491-498.

39. Davis MD, Sheetz MJ, Aiello LP, Milton RC, Danis RP, et al. (2009) Effect of ruboxistaurin on the visual acuity decline associated with long-standing diabetic macular edema. Investigative Ophthalmology and Visual Science 50(1): 1-4.

40. Lafuente M, Ortín L, Argente M, Guindo JL, López B, et al. (2017) Combined intravitreal ranibizumab and oral supplementation with docosahexaenoic acid and antioxidants for diabetic macular edema: Two-year randomized single-blind controlled trial results. RETINA 37(7): 1277-1286.

41. Moschos MM, Dettoraki M, Tsatsos M, Kitsos G, Kalogeropoulos C (2017) Effect of carotenoids dietary supplementation on macular function in diabetic patients. Eye and visión 4(23).

42. Elman MJ, Qin H, Aiello LP, Beck RW, Bressler NM, et al. (2012) Intravitreal ranibizumab for diabetic macular edema with prompt versus deferred laser treatment: three-year randomized trial results. Ophthalmology 119(11): 2312-2318.

43. Campos PR, Rubio SC, García Guisado DM, Díaz Luque MJ (2018) Aflibercept for clinically significant diabetic macular edema: 12-month results in daily clinical practice. Clinical ophthalmology 12: 99-104.

44. Shimizu N, Oshitari T, Tatsumi T, Takatsuna Y, Arai M, et al. (2017) Comparisons of efficacy of intravitreal aflibercept and ranibizumab in eyes with diabetic macular edema. BioMed Research International. 